Review

\title{
Biofilms: The Stronghold of Legionella pneumophila
}

\author{
Mena Abdel-Nour ${ }^{1,2,3}$, Carla Duncan ${ }^{1}$, Donald E. Low ${ }^{2,3, \dagger}$ and Cyril Guyard ${ }^{1,2,3, *}$
}

1 Ontario Agency for Health Protection and Promotion (OAHPP), Toronto, ON M9P 3T1, Canada;

E-Mails: mena_abdelnour@hotmail.com (M.A.-N.); carla.duncan@oahpp.ca (C.D.)

2 Department of Laboratory Medicine and Pathobiology, University of Toronto, Toronto, ON M5S 1A8, Canada

3 Mount Sinai Hospital, Toronto, ON M5G 1X5, Canada

$\dagger$ Deceased.

* Author to whom correspondence should be addressed; E-Mail: cyril.guyard@gmail.com; Tel.: +1-416-880-1339; Fax: +1-416-235-6281.

Received: 9 August 2013; in revised form: 7 September 2013 / Accepted: 14 October 2013 / Published: 31 October 2013

\begin{abstract}
Legionellosis is mostly caused by Legionella pneumophila and is defined as a severe respiratory illness with a case fatality rate ranging from $5 \%$ to $80 \%$. L. pneumophila is ubiquitous in natural and anthropogenic water systems. L. pneumophila is transmitted by inhalation of contaminated aerosols produced by a variety of devices. While L. pneumophila replicates within environmental protozoa, colonization and persistence in its natural environment are also mediated by biofilm formation and colonization within multispecies microbial communities. There is now evidence that some legionellosis outbreaks are correlated with the presence of biofilms. Thus, preventing biofilm formation appears as one of the strategies to reduce water system contamination. However, we lack information about the chemical and biophysical conditions, as well as the molecular mechanisms that allow the production of biofilms by L. pneumophila. Here, we discuss the molecular basis of biofilm formation by L. pneumophila and the roles of other microbial species in L. pneumophila biofilm colonization. In addition, we discuss the protective roles of biofilms against current L. pneumophila sanitation strategies along with the initial data available on the regulation of L. pneumophila biofilm formation.
\end{abstract}

Keywords: Legionella pneumophila; legionellosis; amoeba; prozoa; multispecies biofilms 


\section{Introduction}

The Gram-negative bacterium Legionella pneumophila is responsible for the majority of legionellosis cases and is a significant contributor of community acquired, and hospital acquired pneumonia with a case fatality rate ranging from $5 \%$ to $80 \%$ [1,2]. L. pneumophila is an aquatic pathogen that is ubiquitously found in nature, in both anthropogenic structures and in environmental waters [3-7]. In vitro, L. pneumophila is able to produce monospecies biofilms (Figure 1) that is believed to contain an extracellular matrix [8,9]. In the environment, L. pneumophila can be found in several different habitats, including multispecies biofilms. In naturally occurring multispecies biofilms, the colonization with L. pneumophila can be influenced by several other species of microorganisms [10,11]. Of these microorganisms, protozoa are arguably one of the most important in determining L. pneumophila persistence, as the pathogen uses protozoa to replicate intracellularly [12]. Co-evolution with multiple species of protozoa has resulted in the development of mechanisms that allow L. pneumophila to occupy a very broad host range, and to infect human cells [13-15].

Figure 1. Monospecies biofilm of L. pneumophila labelled with the DNA stain Syto62. Scale bar represents $100 \mu \mathrm{m}$.
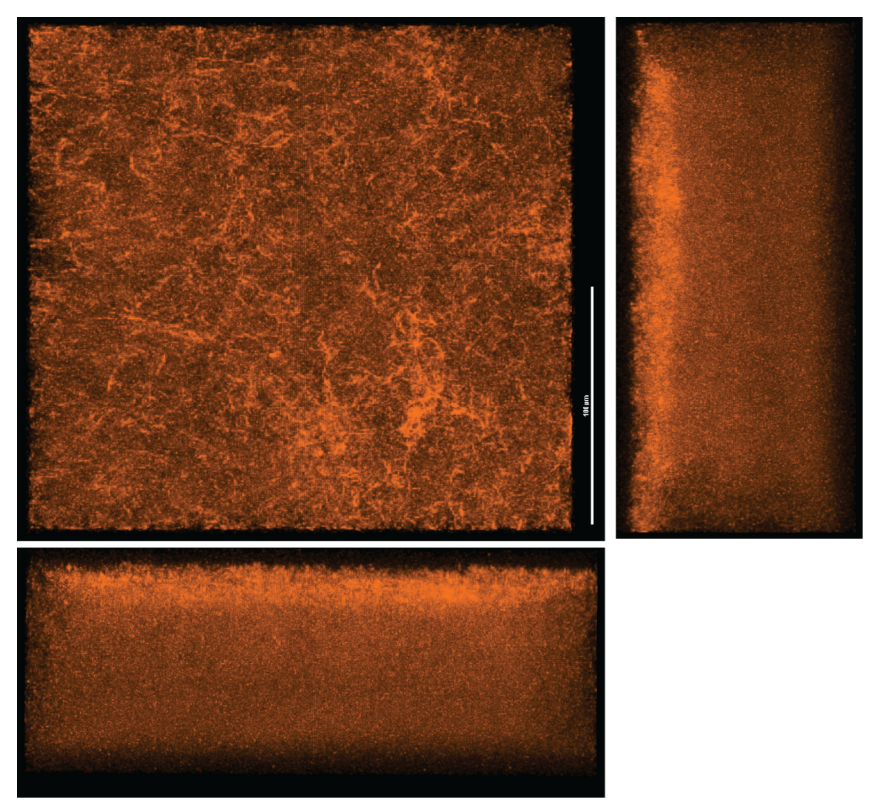

Recent reports suggest that the growth of Legionella in biofilms may lead to enhanced virulence. L. pneumophila isolates from serogroups 1, 10, and 12 that were collected from biofilms were more cytotoxic towards amoeba than reference outbreak and worldwide epidemic strains [16]. Moreover, initial data suggest that biofilm-derived Legionella pneumophila evades the innate immune response in macrophages [17]. As legionellosis is not transmitted from person to person, insights into the ecology of L. pneumophila may yield information that can be used to prevent the colonization of anthropogenic systems by L. pneumophila. In light of recent discoveries, this review intends to provide an overview of the findings on biofilm production and colonization by L. pneumophila. 


\section{Protozoa and L. pneumophila Biofilm Formation}

Protozoa play a crucial role in the lifecycle of Legionella species as they provide a habitat for their environmental survival and replication [12,18-21]. In biofilm communities, several amoeba species have been found associated with L. pneumophila [22]. To feed, protozoan species often graze on bacteria present in multispecies biofilms, a phenomenon that L. pneumophila exploits in order to replicate (Figure 2) [23,24]. As a consequence, the presence of protozoa in anthropogenic water sources has been deemed a risk factor for L. pneumophila outbreaks [23]. In fact, the amount of L. pneumophila in biofilms is directly correlated with the biomass of protozoa [25]. This is in accordance with in vitro models showing that the presence of amoeba species promotes the biofilm formation of L. pneumophila on pins of "inverse" microtiter plates [26]. L. pneumophila is also capable of growing off the debris from dead amoebae, thus, amoeba may also encourage the replication of L. pneumophila indirectly [27]. Floating biofilms, also contain protozoa in association with L. pneumophila suggesting that L. pneumophila may attach to protozoa in floating biofilms in the absence of available abiotic surfaces [28,29]. In addition to the role of protozoa as a means of replication, the intracellular stage of L. pneumophila provides protection from environmental stressors [30,31] including biocides used to disinfect water systems [32,33]. Indeed, biofilms produced with L. pneumophila in the presence of thermotolerant amoebae allow L. pneumophila to persist after heat treatment [34], demonstrating that amoebae can provide a protective niche for L. pneumophila [30].

Figure 2. A. castellanii infected with L. pneumophila expressing green fluorescent protein. Scale bar represents $10 \mu \mathrm{m}$.

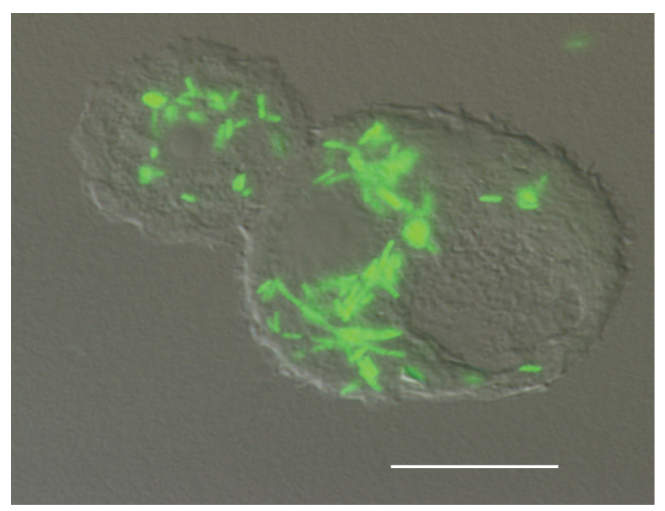

\section{Physiochemical Determinants in L. pneumophila Biofilm Formation and Colonization}

Attachment is the process of one object adhering to another, and when producing surface associated biofilms, attachment of L. pneumophila serves as an initial crucial step, whether it is on biotic or abiotic surfaces. Once bacteria are attached to a substratum and a biofilm is formed, the process of spreading and persisting within a new area is defined as colonization. Although L. pneumophila can often be found attached to various surfaces in the environment, colonization of existing biofilms in addition to attachment to abiotic substrates is determined by a wide variety of parameters (Figure 3) [35]. One important factor that governs the adherence of L. pneumophila in anthropogenic water systems is the composition of the surface material to which the bacteria are adhering [36]. L. pneumophila can adhere well to several different plastics that are commonly used in plumbing, whereas copper inhibits its 
attachment [36-38]. It remains unclear however whether this is due to differences in surface and L. pneumophila interactions or because different plumbing materials select for different pioneering species, which establishes the initial biofilm and L. pneumophila colonizes afterwards.

Figure 3. L. pneumophila (denoted by Lpn and shown in orange) replicate within environmental protozoa. Uptake within protozoan hosts is promoted by the presence of other amoebae and bacterial species such as $P$. aeruginosa (top left) and by environmental cues such as quorum sensing (green hexagons, top right). Environmental cues can also influence changes in L. pneumophila cell metabolism that favour biofilm production and colonization, which may occur following replication within protozoa or independently of protozoa infection (middle). Other microbial species such as $P$. aeruginosa (Lpn antagonistic microbial species, brown) can inhibit L. pneumophila colonization (bottom left). The presence of other microorganisms such as $K$. pneumoniae alleviates the inhibitory effect of $P$. aeruginosa (Lpn permissive microbial species, blue) and allows L. pneumophila to be incorporated within biofilms. L. pneumophila produces a surfactant (brown circles), which is toxic to other Legionella species (red), and may therefore prevent incorporation of these bacteria within biofilms. Physio-chemical parameters such as divalent cations (grey circles) can favour L. pneumophila biofilm colonization while other factors such as the presence of nanoparticles and copper (blue circles) can hinder L. pneumophila colonization.

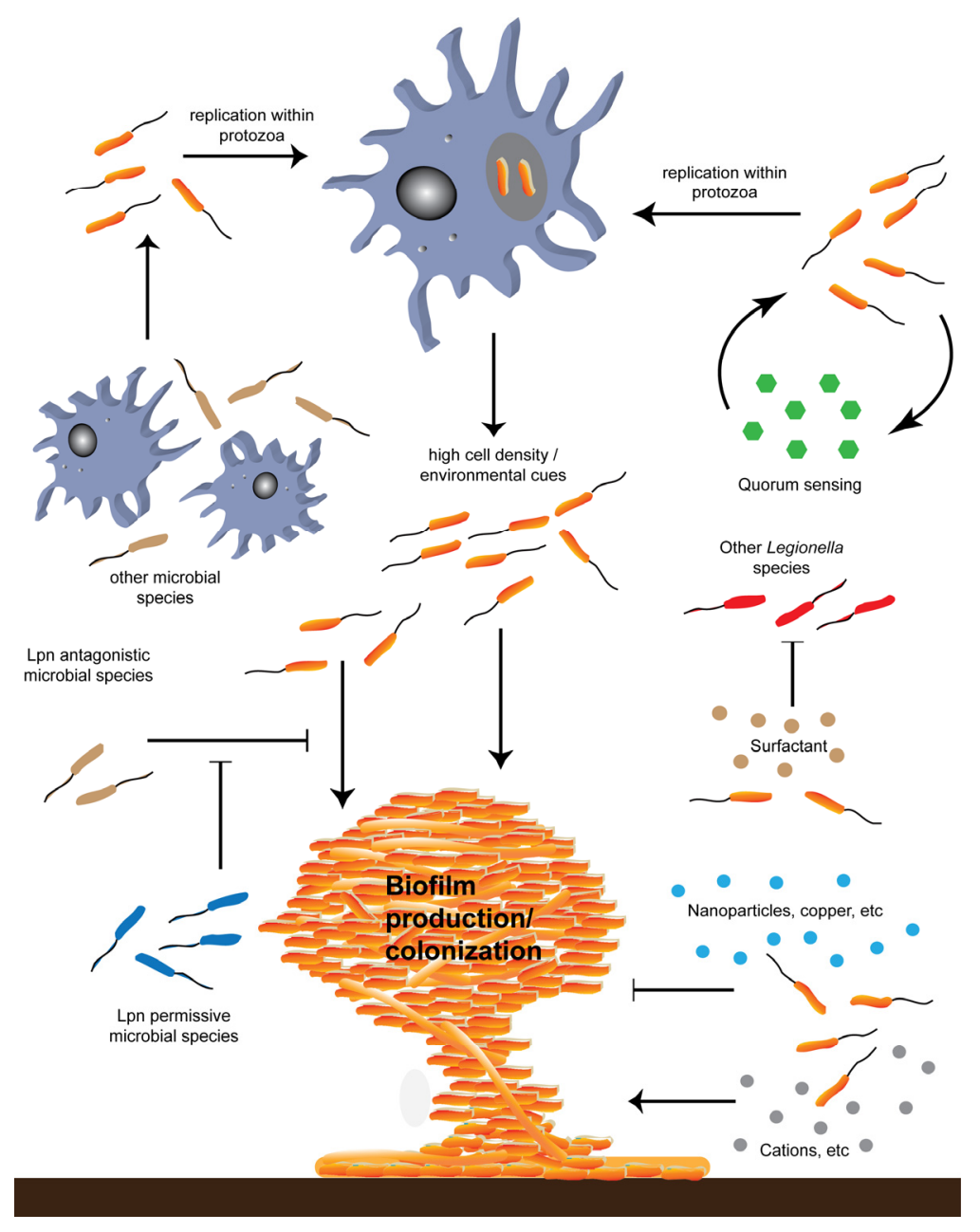


Cations are implicated in the attachment of bacteria to different substrata, and can contribute to biofouling [39]. Similarly, both calcium and magnesium were demonstrated to facilitate the attachment of L. pneumophila to abiotic surfaces [40]. Elevated zinc, magnesium, and manganese levels are correlated with increased L. pneumophila contamination and zinc increases the ability of L. pneumophila to bind to host cells such as human lung epithelial cells, suggesting that cations may increase the attachment of L. pneumophila to biotic surfaces in addition to abiotic substrata [41-43]. Interestingly as it pertains to the cation dependent attachment of L. pneumophila, an orthologue of the Pseudomonas fluorescens calcium-dependent cyclic-diGMP regulated protease LapG was identified in L. pneumophila. LapG regulates biofilm formation of Pseudomonas fluorescens by cleaving the surface adhesin LapA required for biofilm formation [44,45].

In addition to the presence of cations, the availability of carbon favours the colonization of biofilms with L. pneumophila, presumably because it provides nutrients for the bacteria to replicate [46]. Notably, the increase in biofilm production due to organic-carbon has only been reported at $20{ }^{\circ} \mathrm{C}$, suggesting that carbon may only influence biofilm production at certain temperatures [47]. Temperature is also an important determinant for L. pneumophila biofilm colonization. Studies have shown that heating water above $55^{\circ} \mathrm{C}$ can reduce the detectable amount of L. pneumophila in water systems, even in the presence of organic carbon sources, however this may be due to a decrease in other biofilm species which may serve as a platform for L. pneumophila colonization [48,49].

Static and flow conditions of water play important roles in the biofilm formation and biofilm colonization with L. pneumophila in water systems. Stagnation of water in distribution systems seems to favour colonization with L. pneumophila [50]. Moreover, Legionnaires' disease cases have been linked to stagnant water in hospital settings [51]. In accordance with these data, a constant flow in anthropogenic water can decrease the presence of $L$. pneumophila through the use of Venturi systems by preventing the attachment of the bacteria to surfaces [52]. However, biofilms in aquatic environments can persist under turbulent flow conditions [53,54] and maintain a population of L. pneumophila [55]. To explain the persistence of L. pneumophila under turbulent flow, it was proposed that the bacteria can localize to the sediment where it is less affected by turbulence [56,57]. The settling of L. pneumophila in environmental sediments, which was recently linked to quorum sensing, will be discussed below [58,59].

Although the majority of L. pneumophila biofilm formation/colonization research has been focused on determining the physiochemical parameters that allow L. pneumophila to colonize and form biofilms, little is known regarding the L. pneumophila molecular factors that contribute directly to this process. The Legionella collagen-like protein ( $\mathrm{Lcl}$ ) was initially identified as an adhesin required for infection of protozoa and macrophages [60]. Subsequently, Lcl was found to be an important mediator of L. pneumophila biofilm formation [61]. Lcl facilitates biofilm production by promoting attachment to abiotic substrates as well as cell-cell/cell-matrix interactions [62]. Type IV pili was also implicated in L. pneumophila biofilm colonization based in its role in adherence to protozoan cells [63]. However, a site directed type IV pili mutant was shown to colonize biofilms of other organisms as well as wild-type bacteria [64]. In addition to surface exposed adhesins, the twin arginine transport (Tat) secretion system has also been implicated in biofilm formation. Deletion of the tat $B$ and tat $C$ genes resulted in a significant reduction in biofilm formation, however the specific role that this secretion system plays is unknown [65]. 


\section{Regulation of L. pneumophila Endogenous Factors that May Influence Biofilm Colonization}

For L. pneumophila, as well as for other microorganisms, biofilm formation is an environmental response that can promote survival. L. pneumophila, like many other microorganisms, responds to environmental cues, which can greatly influence biofilm formation and or colonization (Figure 3). One important environmental prompt is iron, which has important roles in the growth of many organisms, and can influence L. pneumophila replication [66]. The addition of lactoferrin, an iron chelator, can directly kill L. pneumophila demonstrating the importance of iron in L. pneumophila viability [67]. Furthermore, bacterial ferrous iron transport promotes the intracellular replication of L. pneumophila in protozoa, which may influence multispecies biofilm colonization [68]. Iron is also required for the production of melanin and it is believed that deletion of the $l b t A$ and $l b t B$ genes, which encode iron siderophores prevent growth within aquatic biofilms [66]. Interestingly, although iron is essential for biofilm formation, high iron concentrations can inhibit biofilm formation, yet to date the reasons for this are unknown [8].

The ability of bacteria to monitor and respond to cell density is known as quorum sensing and it is a crucial process during biofilm production. Among quorum sensing molecules, $\alpha$-hydroxy ketones (AHKs) have been identified in L. pneumophila, and are similar to the AHKs produced by Vibrio cholera [69,70]. Although there is currently no evidence that AHKs regulate L. pneumophila biofilm production directly, these molecules regulate a wide variety of traits that may influence L. pneumophila biofilm production/colonization indirectly, including virulence (infection of protozoan hosts in multispecies biofilms), extracellular filament production, and sedimentation through the lqs gene cluster, which encodes for the AHK synthase LqsA, the AHK sensor LqsS and the response regulator LqsR $[58,71,72]$. In addition to the products of this gene cluster, an orphan sensor kinase named LqsT regulates competence, a process that is correlated to biofilm formation in other species [59].

The second-messenger molecule cyclic di-GMP (c-di-GMP) is also an important signaling system that allows bacteria to respond to environmental changes [73] L. pneumophila has 22 predicted genes related to c-di-GMP production, degradation and/or recognition [74]. One of these genes, lpg1057, was found to encode an enzyme responsible for the production of cyclic di-GMP which promotes biofilm formation, and is the only c-di-GMP related gene to date found to directly influence monospecies biofilm production of L. pneumophila [75]. In response to amino acid starvation, the alarmone guanosine tetraphosphate (ppGpp) can also regulate L. pneumophila gene expression [76]. Although the ppGpp system is mainly linked to the regulation of virulence related traits, this system may indirectly affect environmental biofilm colonization by influencing L. pneumophila-amoeba interactions. In addition, sensitivity to ppGpp signaling requires the sigma factor RpoS [77]. RpoS in turn influences LqsR expression, suggesting that virulence related traits regulated by AHKs require multiple environmental signals [78]. In parallel with the ppGpp-RpoS regulation of virulence, downstream is the two-component system LetA/LetS [79]. The LetA/LetS system relieves the repression of virulence related genes by the RNA binding protein CsrA [80]. Despite the initially suspected roles of these transcriptional regulators in surface attachment and biofilm formation, none of the mutants lacking rpoS, letA or csrA were affected in biofilm formation in the L. pneumophila strain JR32 [26]. Of the known L. pneumophila sigma factors, to date only the flagellar sigma factor FliA has been implicated in the regulation of biofilm production and deletion of fliA results in a decrease in 
biofilm formation in JR32, however it is unclear what downstream or upstream factors are involved in this process [26].

Temperature was mentioned above as being an important determinant for biofilm colonization [48,49]. In addition, temperature can regulate the properties of the biofilms produced by L. pneumophila [81]. In vitro, at $37-42{ }^{\circ} \mathrm{C}$, monospecies biofilms are mycelial mat-like and are composed of filamentous bacteria whereas biofilms produced at $25^{\circ} \mathrm{C}$ are thinner and made up of rod shaped cells [81]. These findings coincide with other studies demonstrating that the filamentation of L. pneumophila is regulated by temperature [82]. Filamentous growth occurs in other bacterial species to increase fitness against adverse environmental conditions [83]. In turn, intracellular filamentatous L. pneumophila can produce progeny more efficiently than short rod forms [84]. Furthermore, the length of L. pneumophila cells has been linked to ppGpp signalling [80]. In vitro, biofilms produced at $37{ }^{\circ} \mathrm{C}$ are much more robust than at $25^{\circ} \mathrm{C}$ [26], and interestingly biofilms produced at $25^{\circ} \mathrm{C}$ are more adherent [81]. In addition, the production of the L. pneumophila type II secretion system, and type IV pili are temperature regulated, and may influence attachment at different temperatures [85].

\section{The Role of Non-Protozoa Microbial Species in L. pneumophila Biofilm Colonization}

Environmental biofilms often contain several different bacterial species [86]. These bacterial species may promote the persistence of L. pneumophila in biofilms, while other species inhibit L. pneumophila's colonization (Figure 3). For example, Flavobacterum breve and cyanobacterial species can promote L. pneumophila growth and colonization in biofilms by providing a source of nutrients $[87,88]$. In vitro, the growth of L. pneumophila is necrotrophic when heat killed Pseudomonas putida bacteria are given as a nutrient source, however heat killed Gram-positive organisms such Bacillus subtilis and Lactobacillus plantarum did not alter the growth of L. pneumophila, suggesting that L. pneumophila is capable of replicating without the presence of protozoan species, and that necrotrophic growth of L. pneumophila is restricted to certain microbial species [27].

Summer seasons, which coincide with legionellosis outbreaks, favour the proliferation of L. pneumophila in cooling tower microbial populations while other Legionella species decrease in number [89]. Based on this shift in abundance it has been hypothesized that L. pneumophila may inhibit the growth of other Legionella species. In fact, L. pneumophila produces a surfactant secreted by the protein TolC, which is toxic to other Legionella species, but has no effect on Pseudomonas aeruginosa, Klebsiella pneumoniae and Listeria monocytogenes [90]. Therefore it is tempting to speculate that L. pneumophila may influence the growth of other Legionella species in their natural environment.

One of the most studied bacteria that can influence L. pneumophila's biofilm colonization ability is $P$. aeruginosa. Although there is a body of evidence suggesting that L. pneumophila can coexist in biofilms with $P$. aeruginosa, these studies were performed with inoculums from natural environmental sources, which may contain several different bacterial species $[11,38]$. In contrast to these studies, monospecies biofilms with $P$. aeruginosa were shown to prevent $L$. pneumophila colonization [26,91]. This phenomenon may be mediated by acylhomoserine lactones (AHLs) produced by P. aeruginosa as these AHLs not only inhibit the growth of L. pneumophila but also its biofilm production [92]. Furthermore, specific AHLs produced by P. aeruginosa can downregulate Lcl production, which is essential for biofilm formation in L. pneumophila [62]. Interestingly, the in vitro inhibition of 
L. pneumophila colonization by $P$. aeruginosa is alleviated if $K$. pneumoniae is present in the produced biofilm [91]. In fact complex multispecies biofilms that contain both $P$. aeruginosa and $K$. pneumoniae are permissive for L. pneumophila colonization [28]. The presence of amoeba seems to also effect whether $P$. aeruginosa is antagonistic to L. pneumophila colonization, as biofilms which contain both Acanthamoeba castellanii and P. aeruginosa, increase the uptake of L. pneumophila within A. castellanii, and the colonization of L. pneumophila in biofilms [93].

\section{The Resistance of L. pneumophila Containing Biofilms to Biocides}

There is a great interest in improving methods for disinfecting L. pneumophila containing biofilms because of the ongoing threat to human health posed by these organisms in anthropogenic water sources. Due to the intracellular lifestyle of L. pneumophila within protozoa, however, it is difficult to tease out whether the resistance of L. pneumophila in environmental biofilms is due to the biofilm structure, its association with amoeba or both. It is however evident that environmental L. pneumophila found in biofilms are extremely resilient to treatment with biocides [94]. L. pneumophila exposed to environmental stresses and/or found within biofilms can enter a viable but non-culturable (VBNC) state, and treatment of water systems with biocides can make L. pneumophila enter the VBNC state [95]. This property makes the accurate assessment of the contamination levels with L. pneumophila cumbersome since it requires the co-culturing of L. pneumophila with amoeba to lift the VBNC state [96].

Recently, nanoparticles have been suggested to be powerful tools to prevent L. pneumophila biofilm formation, as nanoparticles are able to disrupt L. pneumophila-amoebae interactions and biofilm structure [97,98]. Nanoparticles can also effectively clear L. pneumophila from mixed species biofilms and appear to be an attractive treatment option for disinfecting anthropogenic water sources [99]. The most common biocides used to control water-borne pathogens are generally chlorine derivatives, and chlorine derivatives are more efficacious than UV for disinfecting L. pneumophila [100-102]. Yet chloramine, one of the most potent chlorine derivative biocides, does not completely eradicate L. pneumophila from aquatic biofilms $[103,104]$.

The location of the biofilm can also play a role in resistance to disinfection strategies. This is particularly the case for biofilms formed in sediments, which provide protection to L. pneumophila from UV radiation [105]. Furthermore, L. pneumophila bacteria grown on a solid surface are more resistant to killing by iodine than bacteria grown in broth, suggesting that there are metabolic differences between surface associated and planktonic phase bacteria [106]. This is consistent with data suggesting that sessile and planktonic L. pneumophila in biofilms have different gene expression profiles [8].

\section{Conclusions}

L. pneumophila is an environmental pathogen, and understanding the ecology of this pathogen can help to determine methods for preventing its environmental dissemination and the transmission of legionellosis. There are a multitude of factors that can influence whether L. pneumophila produces biofilms, and likely many more that remain to be uncovered. Although there have been significant advances in the understanding L. pneumophila biofilm formation and colonization in the last several 
years, there is much that remains unknown. The presence of other microbial species, physiochemical parameters, and L. pneumophila gene regulation are all factors that could potentially be exploited to prevent colonization of L. pneumophila in anthropogenic systems. The physiochemical parameters, which favour biofilm, formation is a topic of great interest. Research has yielded insight into factors, which could potentially limit L. pneumophila growth and may be useful for the prevention of legionellosis. One area of increasing interest is the role of other bacterial species in L. pneumophila biofilm production, and the mechanism with which certain species promote L. pneumophila growth while other species inhibit it. Another question that remains to be answered is to what extent the intracellular lifestyle contributes to L. pneumophila biofilm resistance to disinfection in situ. Finally, there is still much unknown about the endogenous factors that L. pneumophila utilizes to facilitate biofilm formation, such as what L. pneumophila quorum sensing systems regulate biofilm formation and what other factors are involved, (for example an extracellular matrix has been found in L. pneumophila monospecies biofilms but has yet to be characterized [8]). Ultimately, this research can yield valuable information that can lead to translational research for prevention and protection against L. pneumophila infections.

\section{Acknowledgments}

This work was supported by the Ontario Agency for Health protection and Promotion, and the Canadian Institutes of Health Research (MOP-102514).

\section{Conflicts of Interest}

The authors declare no conflict of interest.

\section{References}

1. Carratala, J.; Fernandez-Sabe, N.; Ortega, L.; Castellsague, X.; Roson, B.; Dorca, J.; Fernandez-Aguera, A.; Verdaguer, R.; Martinez, J.; Manresa, F.; et al. Outpatient care compared with hospitalization for community-acquired pneumonia: A randomized trial in low-risk patients. Ann. Intern. Med. 2005, 142, 165-172.

2. Yu, V.L.; Plouffe, J.F.; Pastoris, M.C.; Stout, J.E.; Schousboe, M.; Widmer, A.; Summersgill, J.; File, T.; Heath, C.M.; Paterson, D.L.; et al. Distribution of Legionella species and serogroups isolated by culture in patients with sporadic community-acquired Legionellosis: An international collaborative survey. J. Infect. Dis. 2002, 186, 127-128.

3. Bentham, R.H.; Broadbent, C.R. A model for autumn outbreaks of Legionnaires' disease associated with cooling towers, linked to system operation and size. Epidemiol. Infect. 1993, 111, 287-295.

4. Fields, B.S.; Benson, R.F.; Besser, R.E. Legionella and Legionnaires' disease: 25 years of investigation. Clin. Microbiol. Rev. 2002, 15, 506-526.

5. Feazel, L.M.; Baumgartner, L.K.; Peterson, K.L.; Frank, D.N.; Kirk Harris, J.; Pace, N.R. Opportunistic pathogens enriched in showerhead biofilms. Proc. Natl. Acad. Sci. USA 2009, 106, 16393-16398. 
6. McEvoy, M.; Batchelor, N.; Hamilton, G.; MacDonald, A.; Faiers, M.; Sills, A.; Lee, J.; Harrison, T. A cluster of cases of Legionnaires' disease associated with exposure to a spa pool on display. Commun. Dis. Public Health 2000, 3, 43-45.

7. Wadowsky, R.M.; Yee, R.B.; Mezmar, L.; Wing, E.J.; Dowling, J.N. Hot water systems as sources of Legionella pneumophila in hospital and nonhospital plumbing fixtures. Appl. Environ. Microbiol. 1982, 43, 1104-1110.

8. Hindre, T.; Bruggemann, H.; Buchrieser, C.; Hechard, Y. Transcriptional profiling of Legionella Pneumophila biofilm cells and the influence of iron on biofilm formation. Microbiology 2008, 154, 30-41.

9. Pecastaings, S.; Berge, M.; Dubourg, K.M.; Roques, C. Sessile Legionella pneumophila is able to grow on surfaces and generate structured monospecies biofilms. Biofouling 2010, 26, 809-819.

10. Kwon, S.; Moon, E.; Kim, T.; Hong, S.; Park, H. Pyrosequencing demonstrated complex microbial communities in a membrane filtration system for a drinking water treatment plant. Microbes Environ. 2011, 26, 149-155.

11. Murga, R.; Forster, T.S.; Brown, E.; Pruckler, J.M.; Fields, B.S.; Donlan, R.M. Role of biofilms in the survival of Legionella pneumophila in a model potable-water system. Microbiology 2001, 147, 3121-3126.

12. Rowbotham, T.J. Pontiac fever, ameobae and Legionllae. Lancet 1981, 317, 40-41.

13. Molmeret, M.; Horn, M.; Wagner, M.; Santic, M.; Abu Kwaik, Y. Amoebae as training grounds for intracellular bacterial pathogens. Appl. Environ. Microbiol. 2005, 71, 20-28.

14. Steinert, M.; Hentschel, U.; Hacker, J. Legionella pneumophila: An aquatic microbe goes astray. FEMS Microbiol. Rev. 2002, 26, 149-162.

15. Yan, L.; Cerny, R.L.; Cirillo, J.D. Evidence that hsp90 is involved in the altered interactions of Acanthamoeba castellanii variants with bacteria. Eukaryotic Cell 2004, 3, 567-578.

16. Chaabna, Z.; Forey, F.; Reyrolle, M.; Jarraud, S.; Atlan, D.; Fontvieille, D.; Gilbert, C. Molecular diversity and high virulence of Legionella pneumophila strains isolated from biofilms developed within a warm spring of a thermal spa. BMC Microbiol. 2013, 13, 17.

17. Abu Khweek, A.; Fernandez Davila, N.S.; Caution, K.; Akhter, A.; Abdulrahman, B.A.; Tazi, M.; Hassan, H.; Novotny, L.A.; Bakaletz, L.O.; Amer, A.O. Biofilm-derived Legionella pneumophila evades the innate immune response in macrophages. Front. Cell Infect. Microbiol. 2013, 3, 18.

18. Barker, J.; Lambert, P.A.; Brown, M.R. Influence of intra-amoebic and other growth conditions on the surface properties of Legionella pneumophila. Infect. Immun. 1993, 61, 3503-3510.

19. Newsome, A.L.; Farone, M.B.; Berk, S.G.; Gunderson, J.H. Free living amoebae as opportunistic hosts for intracellular bacterial parasites. J. Eukaryot. Microbiol. 2001, 48, 13S-14S.

20. Rowbotham, T.J. Isolation of Legionella pneumophila from clinical specimens via amoebae, and the interaction of those and other isolates with amoebae. J. Clin. Pathol. 1983, 36, 978-986.

21. Skinner, A.R.; Anand, C.M.; Malic, A.; Kurtz, J.B. Acanthamoebae and environmental spread of Legionella pneumophila. Lancet 1983, 2, 289-290.

22. Taylor, M.; Ross, K.; Bentham, R. Legionella, protozoa, and biofilms: Interactions within complex microbial systems. Microb. Ecol. 2009, 58, 538-547. 
23. Valster, R.M.; Wullings, B.A.; van der Kooij, D. Detection of protozoan hosts for Legionella pneumophila in engineered water systems by using a biofilm batch test. Appl. Environ. Microbiol. 2010, 76, 7144-7153.

24. Valster, R.M.; Wullings, B.A.; van den Berg, R.; van der Kooij, D. Relationships between free-living protozoa, cultivable Legionella spp., and water quality characteristics in three drinking water supplies in the caribbean. Appl. Environ. Microbiol. 2011, 77, 7321-7328.

25. Liu, R.; Yu, Z.; Guo, H.; Liu, M.; Zhang, H.; Yang, M. Pyrosequencing analysis of eukaryotic and bacterial communities in faucet biofilms. Sci. Total Environ. 2012, 435-436, 124-131.

26. Mampel, J.; Spirig, T.; Weber, S.S.; Haagensen, J.A.; Molin, S.; Hilbi, H. Planktonic replication is essential for biofilm formation by Legionella pneumophila in a complex medium under static and dynamic flow conditions. Appl. Environ. Microbiol. 2006, 72, 2885-2895.

27. Temmerman, R.; Vervaeren, H.; Noseda, B.; Boon, N.; Verstraete, W. Necrotrophic growth of Legionella pneumophila. Appl. Environ. Microbiol. 2006, 72, 4323-4328.

28. Declerck, P.; Behets, J.; van Hoef, V.; Ollevier, F. Replication of Legionella pneumophila in floating biofilms. Curr. Microbiol. 2007, 55, 435-440.

29. Hsu, B.; Huang, C.; Chen, J.; Chen, N.; Huang, J. Comparison of potentially pathogenic free-living amoeba hosts by Legionella spp. in substrate-associated biofilms and floating biofilms from spring environments. Water Res. 2011, 45, 5171-5183.

30. Atlas, R.M. Legionella: From environmental habitats to disease pathology, detection and control. Environ. Microbiol. 1999, 1, 283-293.

31. Hoffman, P.S. Invasion of eukaryotic cells by Legionella pneumophila: A common strategy for all hosts? Can. J. Infect. Dis. 1997, 8, 139-146.

32. Donlan, R.M.; Forster, T.; Murga, R.; Brown, E.; Lucas, C.; Carpenter, J.; Fields, B. Legionella pneumophila associated with the protozoan Hartmannella vermiformis in a model multi-species biofilm has reduced susceptibility to disinfectants. Biofouling 2005, 21, 1-7.

33. Thomas, V.; Bouchez, T.; Nicolas, V.; Robert, S.; Loret, J.F.; Levi, Y. Amoebae in domestic water systems: Resistance to disinfection treatments and implication in Legionella persistence. J. Appl. Microbiol. 2004, 97, 950-963.

34. Storey, M.V.; Winiecka-Krusnell, J.; Ashbolt, N.J.; Stenstrom, T. The efficacy of heat and chlorine treatment against thermotolerant Acanthamoebae and Legionellae. Scand. J. Infect. Dis. 2004, 36, 656-662.

35. Wright, J.B.; Ruseska, I.; Athar, M.A.; Corbett, S.; Costerton, J.W. Legionella pneumophila grows adherent to surfaces in vitro and in situ. Infect. Control Hosp. Epidemiol. 1989, 10, 408-415.

36. Rogers, J.; Dowsett, A.B.; Dennis, P.J.; Lee, J.V.; Keevil, C.W. Influence of plumbing materials on biofilm formation and growth of Legionella pneumophila in potable water systems. Appl. Environ. Microbiol. 1994, 60, 1842-1851.

37. Rogers, J.; Dowsett, A.B.; Dennis, P.J.; Lee, J.V.; Keevil, C.W. Influence of temperature and plumbing material selection on biofilm formation and growth of Legionella pneumophila in a model potable water system containing complex microbial flora. Appl. Environ. Microbiol. 1994, $60,1585-1592$. 
38. Moritz, M.M.; Flemming, H.; Wingender, J. Integration of Pseudomonas aeruginosa and Legionella pneumophila in drinking water biofilms grown on domestic plumbing materials. Int. J. Hyg. Environ. Health 2010, 213, 190-197.

39. Geesey, G.G.; Wigglesworth-Cooksey, B.; Cooksey, K.E. Influence of calcium and other cations on surface adhesion of bacteria and diatoms: A review. Biofouling 2000, 15, 195-205.

40. Koubar, M.; Rodier, M.H.; Frere, J. Involvement of minerals in adherence of Legionella pneumophila to surfaces. Curr. Microbiol. 2013, 66, 437-442.

41. Yaradou, D.F.; Raze, D.; Ginevra, C.; Ader, F.; Doleans-Jordheim, A.; Vandenesch, F.; Menozzi, F.D.; Etienne, J.; Jarraud, S. Zinc-dependent cytoadherence of Legionella pneumophila to human alveolar epithelial cells in vitro. Microb. Pathog. 2007, 43, 234-242.

42. Bargellini, A.; Marchesi, I.; Righi, E.; Ferrari, A.; Cencetti, S.; Borella, P.; Rovesti, S. Parameters predictive of Legionella contamination in hot water systems: Association with trace elements and heterotrophic plate counts. Water Res. 2011, 45, 2315-2321.

43. Borella, P.; Montagna, M.T.; Romano-Spica, V.; Stampi, S.; Stancanelli, G.; Triassi, M.; Marchesi, I.; Bargellini, A.; Neglia, R.; Paglionico, N.; et al. Relationship between mineral content of domestic hot water and microbial contamination. J. Trace Elem. Med. Biol. 2003, 17, 37-43.

44. Boyd, C.D.; Chatterjee, D.; Sondermann, H.; O’Toole, G.A. LapG, required for modulating biofilm formation by Pseudomonas fluorescens Pf0-1, is a calcium-dependent protease. J. Bacteriol. 2012, 194, 4406-4414.

45. Chatterjee, D.; Boyd, C.D.; O’Toole, G.A.; Sondermann, H. Structural characterization of a conserved, calcium-dependent periplasmic protease from Legionella pneumophila. J. Bacteriol. 2012, 194, 4415-4425.

46. Giao, M.S.; Wilks, S.A.; Azevedo, N.F.; Vieira, M.J.; Keevil, C.W. Comparison between standard culture and peptide nucleic acid 16S rRNA hybridization quantification to study the influence of physico-chemical parameters on Legionella pneumophila survival in drinking water biofilms. Biofouling 2009, 25, 343-351.

47. Pang, C.M.; Liu, W. Biological filtration limits carbon availability and affects downstream biofilm formation and community structure. Appl. Environ. Microbiol. 2006, 72, 5702-5712.

48. Van der Kooij, D.K.; Veenendaal, H.R.; Scheffer, W.J.H. Biofilm formation and multiplication of Legionella in a model warm water system with pipes of copper, stainless steel and cross-linked polyethylene. Water Res. 2005, 39, 2789-2798.

49. Martinelli, F.; Caruso, A.; Moschini, L.; Turano, A.; Scarcella, C.; Speziani, F. A comparison of Legionella pneumophila occurrence in hot water tanks and instantaneous devices in domestic, nosocomial, and community environments. Curr. Microbiol. 2000, 41, 374-376.

50. Ciesielski, C.A.; Blaser, M.J.; Wang, W.L. Role of stagnation and obstruction of water flow in isolation of Legionella pneumophila from hospital plumbing. Appl. Environ. Microbiol. 1984, 48, 984-987.

51. Fisher-Hoch, S.; Bartlett, C.L.; Tobin, J.O.; Gillett, M.B.; Nelson, A.M.; Pritchard, J.E.; Smith, M.G.; Swann, R.A.; Talbot, J.M.; Thomas, J.A. Investigation and control of an outbreaks of Legionnaires' disease in a district general hospital. Lancet 1981, 1, 932-936. 
52. Makin, T.; Pride, M. Venturi system could be major breakthrough. Health Estate 2010, 64, 27-33.

53. Storey, M.V.; Langmark, J.; Ashbolt, N.J.; Stenstrom, T.A. The fate of Legionellae within distribution pipe biofilms: Measurement of their persistence, inactivation and detachment. Water Sci. Technol. 2004, 49, 269-275.

54. Lehtola, M.J.; Torvinen, E.; Kusnetsov, J.; Pitkanen, T.; Maunula, L.; von Bonsdorff, C.; Martikainen, P.J.; Wilks, S.A.; Keevil, C.W.; Miettinen, I.T. Survival of Mycobacterium avium, Legionella pneumophila, Escherichia coli, and caliciviruses in drinking water-associated biofilms grown under high-shear turbulent flow. Appl. Environ. Microbiol. 2007, 73, 2854-2859.

55. Liu, Z.; Lin, Y.E.; Stout, J.E.; Hwang, C.C.; Vidic, R.D.; Yu, V.L. Effect of flow regimes on the presence of Legionella within the biofilm of a model plumbing system. J. Appl. Microbiol. 2006, $101,437-442$.

56. Fliermans, C.B.; Cherry, W.B.; Orrison, L.H.; Smith, S.J.; Tison, D.L.; Pope, D.H. Ecological distribution of Legionella pneumophila. Appl. Environ. Microbiol. 1981, 41, 9-16.

57. Stout, J.E.; Yu, V.L.; Best, M.G. Ecology of Legionella pneumophila within water distribution systems. Appl. Environ. Microbiol. 1985, 49, 221-228.

58. Tiaden, A.; Spirig, T.; Sahr, T.; Walti, M.A.; Boucke, K.; Buchrieser, C.; Hilbi, H. The autoinducer synthase LqsA and putative sensor kinase lqss regulate phagocyte interactions, extracellular filaments and a genomic island of Legionella pneumophila. Environ. Microbiol. 2010, 12, 1243-1259.

59. Kessler, A.; Schell, U.; Sahr, T.; Tiaden, A.; Harrison, C.; Buchrieser, C.; Hilbi, H. The Legionella pneumophila orphan sensor kinase LqsT regulates competence and pathogen-host interactions as a component of the LAI-1 circuit. Environ. Microbiol. 2013, 15, 646-662.

60. Vandersmissen, L.; de Buck, E.; Saels, V.; Coil, D.A.; Anne, J. A Legionella pneumophila collagen-like protein encoded by a gene with a variable number of tandem repeats is involved in the adherence and invasion of host cells. FEMS Microbiol. Lett. 2010, 306, 168-176.

61. Duncan, C.; Prashar, A.; So, J.; Tang, P.; Low, D.E.; Terebiznik, M.; Guyard, C. Lcl of Legionella pneumophila is an immunogenic GAG binding adhesin that promotes interactions with lung epithelial cells and plays a crucial role in biofilm formation. Infect. Immun. 2011, 6, 2168-2181.

62. Mallegol, J.; Duncan, C.; Prashar, A.; So, J.; Low, D.E.; Terebeznik, M.; Guyard, C. Essential roles and regulation of the Legionella pneumophila collagen-like adhesin during biofilm formation. PLoS One 2012, 7, e46462.

63. Stone, B.J.; Kwaik, Y.A. Expression of multiple pili by Legionella pneumophila: Identification and characterization of a type IV pilin gene and its role in adherence to mammalian and protozoan cells. Infect. Immun. 1998, 66, 1768-1775.

64. Lucas, C.E.; Brown, E.; Fields, B.S. Type IV Pili and Type II secretion play a limited role in Legionella pneumophila biofilm colonization and retention. Microbiology 2006, 152, 3569-3573.

65. De Buck, E.; Maes, L.; Meyen, E.; van Mellaert, L.; Geukens, N.; Anne, J.; Lammertyn, E. Legionella pneumophila Philadelphia-1 tatB and tatC affect intracellular replication and biofilm formation. Biochem. Biophys. Res. Commun. 2005, 331, 1413-1420.

66. Cianciotto, N.P. Iron acquisition by Legionella pneumophila. Biometals 2007, 20, 323-331. 
67. Orsi, N. The antimicrobial activity of lactoferrin: Current status and perspectives. Biometals 2004, 17, 189-196.

68. Robey, M.; Cianciotto, N.P. Legionella pneumophila feoAB promotes ferrous iron uptake and intracellular infection. Infect. Immun. 2002, 70, 5659-5669.

69. Tiaden, A.; Hilbi, H. A-hydroxyketone synthesis and sensing by Legionella and Vibrio. Sensors 2012, 12, 2899-2919.

70. Spirig, T.; Tiaden, A.; Kiefer, P.; Buchrieser, C.; Vorholt, J.A.; Hilbi, H. The Legionella autoinducer synthase LqsA produces an $\alpha$-hydroxyketone signaling molecule. J. Biol. Chem. 2008, 283, 18113-18123.

71. Tiaden, A.; Spirig, T.; Hilbi, H. Bacterial gene regulation by $\alpha$-hydroxyketone signaling. Trends Microbiol. 2010, 18, 288-297.

72. Tiaden, A.; Spirig, T.; Carranza, P.; Bruggemann, H.; Riedel, K.; Eberl, L.; Buchrieser, C.; Hilbi, H. Synergistic contribution of the Legionella pneumophila Lqs genes to pathogen-host interactions. J. Bacteriol. 2008, 190, 7532-7547.

73. McDonough, K.A.; Rodriguez, A. The myriad roles of cyclic AMP in microbial pathogens: From signal to sword. Nat. Rev. Microbiol. 2011, 10, 27-38.

74. Levi, A.; Folcher, M.; Jenal, U.; Shuman, H.A. Cyclic diguanylate signaling proteins control intracellular growth of Legionella pneumophila. mBio 2011, 2, e00316-10.

75. Carlson, H.K.; Vance, R.E.; Marletta, M.A. H-NOX regulation of c-di-GMP metabolism and biofilm formation in Legionella pneumophila. Mol. Microbiol. 2010, 77, 930-942.

76. Hammer, B.K.; Swanson, M.S. Co-ordination of Legionella pneumophila virulence with entry into stationary phase by ppGpp. Mol. Microbiol. 1999, 33, 721-731.

77. Bachman, M.A.; Swanson, M.S. RpoS co-operates with other factors to induce Legionella pneumophila virulence in the stationary phase. Mol. Microbiol. 2001, 40, 1201-1214.

78. Tiaden, A.; Spirig, T.; Weber, S.S.; Bruggemann, H.; Bosshard, R.; Buchrieser, C.; Hilbi, H. The Legionella pneumophila response regulator LqsR promotes host cell interactions as an element of the virulence regulatory network controlled by RpoS and LetA. Cell. Microbiol. 2007, 9, 2903-2920.

79. Hammer, B.K.; Tateda, E.S.; Swanson, M.S. A two-component regulator induces the transmission phenotype of stationary-phase Legionella pneumophila. Mol. Microbiol. 2002, 44, 107-118.

80. Molofsky, A.B.; Swanson, M.S. Legionella pneumophila CsrA is a pivotal repressor of transmission traits and activator of replication. Mol. Microbiol. 2003, 50, 445-461.

81. Piao, Z.; Sze, C.C.; Barysheva, O.; Iida, K.; Yoshida, S. Temperature-regulated formation of mycelial mat-like biofilms by Legionella pneumophila. Appl. Environ. Microbiol. 2006, 72, 1613-1622.

82. Konishi, T.; Yamashiro, T.; Koide, M.; Nishizono, A. Influence of temperature on growth of Legionella pneumophila biofilm determined by precise temperature gradient incubator. J. Biosci. Bioeng. 2006, 101, 478-484.

83. Justice, S.S.; Hunstad, D.A.; Cegelski, L.; Hultgren, S.J. Morphological plasticity as a bacterial survival strategy. Nat. Rev. Microbiol. 2008, 6, 162-168. 
84. Prashar, A.; Bhatia, S.; Tabatabaeiyazdi, Z.; Duncan, C.; Garduno, R.A.; Tang, P.; Low, D.E.; Guyard, C.; Terebiznik, M.R. Mechanism of invasion of lung epithelial cells by filamentous Legionella pneumophila. Cell. Microbiol. 2012, 14, 1632-1655.

85. Liles, M.R.; Viswanathan, V.K.; Cianciotto, N.P. Identification and temperature regulation of Legionella pneumophila genes involved in type IV pilus biogenesis and type II protein secretion. Infect. Immun. 1998, 66, 1776-1782.

86. Messi, P.; Anacarso, I.; Bargellini, A.; Bondi, M.; Marchesi, I.; de Niederhausern, S.; Borella, P. Ecological behaviour of three serogroups of Legionella pneumophila within a model plumbing system. Biofouling 2011, 27, 165.

87. Tison, D.L.; Pope, D.H.; Cherry, W.B.; Fliermans, C.B. Growth of Legionella pneumophila in association with blue-green algae (Cyanobacteria). Appl. Environ. Microbiol. 1980, 39, 456-459.

88. Wadowsky, R.M.; Yee, R.B. Satellite growth of Legionella pneumophila with an environmental isolate of Flavobacterium breve. Appl. Environ. Microbiol. 1983, 46, 1447-1449.

89. Wery, N.; Bru-Adan, V.; Minervini, C.; Delgenes, J.; Garrelly, L.; Godon, J. Dynamics of Legionella spp. and bacterial populations during the proliferation of L. pneumophila in a cooling tower facility. Appl. Environ. Microbiol. 2008, 74, 3030-3037.

90. Stewart, C.R.; Burnside, D.M.; Cianciotto, N.P. The surfactant of Legionella pneumophila is secreted in a TolC-dependent manner and is antagonistic toward other Legionella species. J. Bacteriol. 2011, 193, 5971-5984.

91. Stewart, C.R.; Muthye, V.; Cianciotto, N.P. Legionella pneumophila persists within biofilms formed by Klebsiella pneumoniae, Flavobacterium sp., and Pseudomonas fluorescens under dynamic flow conditions. PLoS One 2012, 7, e50560.

92. Kimura, S.; Tateda, K.; Ishii, Y.; Horikawa, M.; Miyairi, S.; Gotoh, N.; Ishiguro, M.; Yamaguchi, K. Pseudomonas aeruginosa las quorum sensing autoinducer suppresses growth and biofilm production in Legionella species. Microbiology 2009, 155, 1934-1939.

93. Declerck, P.; Behets, J.; Delaedt, Y.; Margineanu, A.; Lammertyn, E.; Ollevier, F. Impact of non-Legionella bacteria on the uptake and intracellular replication of Legionella pneumophila in Acanthamoeba castellanii and Naegleria lovaniensis. Microb. Ecol. 2005, 50, 536-549.

94. Emtiazi, F.; Schwartz, T.; Marten, S.M.; Krolla-Sidenstein, P.; Obst, U. Investigation of natural biofilms formed during the production of drinking water from surface water embankment filtration. Water Res. 2004, 38, 1197-1206.

95. Giao, M.S.; Wilks, S.; Azevedo, N.F.; Vieira, M.J.; Keevil, C.W. Incorporation of natural uncultivable Legionella pneumophila into potable water biofilms provides a protective niche against chlorination stress. Biofouling 2009, 25, 335-341.

96. Alleron, L.; Merlet, N.; Lacombe, C.; Frere, J. Long-term survival of Legionella pneumophila in the viable but nonculturable state after monochloramine treatment. Curr. Microbiol. 2008, 57, 497-502.

97. Raftery, T.D.; Lindler, H.; McNealy, T.L. Altered host cell-bacteria interaction due to nanoparticle interaction with a bacterial biofilm. Microb. Ecol. 2013, 65, 496-503.

98. Stojak, A.R.; Raftery, T.; Klaine, S.J.; McNealy, T.L. Morphological responses of Legionella pneumophila biofilm to nanoparticle exposure. Nanotoxicology 2011, 5, 730-742. 
99. Rogers, J.; Dowsett, A.B.; Keevil, C.W. A paint incorporating silver to control mixed biofilms containing Legionella pneumophila. J. Ind. Microbiol. 1995, 15, 377-383.

100. Liu, Z.; Stout, J.E.; Boldin, M.; Rugh, J.; Diven, W.F.; Yu, V.L. Intermittent use of copper-silver ionization for Legionella control in water distribution systems: A potential option in buildings housing individuals at low risk of infection. Clin. Infect Dis. 1998, 26, 138-140.

101. Schwartz, T.; Hoffmann, S.; Obst, U. Formation of natural biofilms during chlorine dioxide and u.v. disinfection in a public drinking water distribution system. J. Appl. Microbiol. 2003, 95, 591-601.

102. Walker, J.T.; Mackerness, C.W.; Mallon, D.; Makin, T.; Williets, T.; Keevil, C.W. Control of Legionella pneumophila in a hospital water system by chlorine dioxide. J. Ind. Microbiol. 1995, 15, 384-390.

103. Pryor, M.; Springthorpe, S.; Riffard, S.; Brooks, T.; Huo, Y.; Davis, G.; Sattar, S.A. Investigation of opportunistic pathogens in municipal drinking water under different supply and treatment regimes. Water Sci. Technol. 2004, 50, 83-90.

104. Williams, M.M.; Braun-Howland, E. Growth of Escherichia coli in model distribution system biofilms exposed to hypochlorous acid or monochloramine. Appl. Environ. Microbiol. 2003, 69, 5463-5471.

105. Kusnetsov, J.M.; Keskitalo, P.J.; Ahonen, H.E.; Tulkki, A.I.; Miettinen, I.T.; Martikainen, P.J. Growth of Legionella and other heterotrophic bacteria in a circulating cooling water system exposed to ultraviolet irradiation. J. Appl. Microbiol. 1994, 77, 461-466.

106. Cargill, K.L.; Pyle, B.H.; Sauer, R.L.; McFeters, G.A. Effects of culture conditions and biofilm formation on the iodine susceptibility of Legionella pneumophila. Can. J. Microbiol. 1992, 38, 423-429.

(C) 2013 by the authors; licensee MDPI, Basel, Switzerland. This article is an open access article distributed under the terms and conditions of the Creative Commons Attribution license (http://creativecommons.org/licenses/by/3.0/). 